\title{
MAXIMAL SUBLATTICES OF FINITE DISTRIBUTIVE LATTICES. II
}

\author{
IVAN RIVAL
}

\begin{abstract}
Let $L$ be a lattice, $J(L)=\{x \in L \mid x$ join-irreducible in $L\}$ and $M(L)=\{x \in L \mid x$ meet-irreducible in $L\}$. As is well known the sets $J(L)$ and $M(L)$ play a central role in the arithmetic of a lattice $L$ of finite length and particularly, in the case that $L$ is distributive. It is shown that the "quotient set" $Q(L)=\{b / a \mid a \in J(L)$, $b \in M(L), a \leqq b\}$ plays a somewhat analogous role in the study of the sublattices of a lattice $L$ of finite length. If $L$ is a finite distributive lattice, its quotient set $Q(L)$ in a natural way determines the lattice of all sublattices of $L$. By examining the connection between $J(K)$ and $J(L)$, where $K$ is a maximal proper sublattice of a finite distributive lattice $L$, the following is proven: every finite distributive lattice of order $n \geqq 3$ which contains a maximal proper sublattice of order $m$ also contains sublattices of orders $n-m, 2(n-m)$, and $3(n-m)$; and, every finite distributive lattice $L$ contains a maximal proper sublattice $K$ such that either $|K|=|L|-1$ or $|K| \geqq 2 l(L)$, where $l(L)$ denotes the length of $L$.
\end{abstract}

1. Introduction. Let $L$ be a lattice, $J(L)=\{x \in L \mid x$ join-irreducible in $L\}$ and $M(L)=\{x \in L \mid x$ meet-irreducible in $L\}$. As is well known the sets $J(L)$ and $M(L)$ play a central role in the arithmetic of a lattice $L$ of finite length and particularly, in the case that $L$ is distributive. We show (Proposition 1) that the "quotient set" $Q(L)=\{b / a \mid a \in J(L), b \in M(L)$, $a \leqq b\}$ plays a somewhat analogous role in the study of the sublattices of a lattice $L$ of finite length. If $L$ is a finite distributive lattice, its quotient set $Q(L)$ in a natural way determines (Theorem 1) the lattice $\operatorname{Sub}(L)$ of all sublattices of $L$.

By examining (Theorem 2) the connection between $J(K)$ and $J(L)$, where $K$ is a maximal proper sublattice of a finite distributive lattice $L$, we can derive some useful information about the orders of sublattices of finite distributive lattices; namely, every finite distributive lattice of order $n \geqq 3$ which contains a maximal proper sublattice of order $m$ also contains

Received by the editors November 30, 1972.

AMS (MOS) subject classifications (1970). Primary 06A35; Secondary 05A15.

Key words and phrases. Finite distributive lattice, sublattice, maximal proper sublattice, join-irreducible, length.

(C) American Mathematical Society 1974 
sublattices of orders $n-m, 2(n-m)$, and $3(n-m)$; and, every finite distributive lattice $L$ contains a maximal proper sublattice $K$ such that either $|K|=|L|-1$ or $|K| \geqq 2 l(L)$, where $l(L)$ denotes the length of $L$.

The author wishes to thank Barry Wolk for suggesting the proof presented here for Proposition 1. For all terminology not explained here we refer to G. Birkhoff [1].

2. A connection between $Q(L)$ and $\operatorname{Sub}(L)$. Proposition 1 below serves to underline a basic connection between $Q(L)$ and the sublattices of a lattice $L$ of finite length, a connection which, specialized to finite distributive lattices, has been the motivation for the results presented in this paper.

Proposition 1, in fact, is a generalization of Lemma 1 [2]. We shall throughout adopt the abbreviation $\bigcup_{A}[a, b]$ for $\bigcup_{b / a \in A}[a, b]$, where $A \subseteq Q(L)$.

Proposition 1. If $S$ is a sublattice of a lattice $L$ of finite length then $S=L-\bigcup_{A}[a, b]$, for some $A \subseteq Q(L)$.

Proof. We must show that for every $x \in L-S$ there is some $b / a \in Q(L)$ such that $x \in[a, b] \subseteq L-S$. Let us suppose that this does not hold for some $x \in L-S$. Let $A=\{a \in J(L) \mid a \leqq x\}$ and $B=\{b \in M(L) \mid x \leqq b\}$; clearly, $A \neq \varnothing \neq B$ and $\bigvee A=x=\wedge B$. But then by our assumption, for every $a \in A$ and for every $b \in B$ there exists $y_{a}^{b} \in S \cap[a, b]$. Since $L$ is of finite length it is complete; therefore, $\bigvee_{a \in A} \bigwedge_{b \in B} y_{a}^{b} \in S$. On the other hand,

$$
x=\bigvee A=\bigvee a \in A \in \bigwedge_{b \in B} a \leqq \bigvee_{a \in A} \bigwedge_{b \in B} y_{a}^{b} \leqq \bigvee_{a \in A} \bigwedge_{b \in B} b=\wedge B=x,
$$

that is, $\bigvee_{a \in A} \bigwedge_{b \in B} y_{a}^{b}=x \in L-S$, which is a contradiction.

In view of Proposition 1 it is natural to classify sublattices of a lattice $L$ of finite length in terms of subsets of $Q(L)$. Indeed, for $A \subseteq Q(L)$ we define $\mathrm{Cl}(A)=\left\{y|x \in Q(L)|[x, y] \subseteq \bigcup_{A}[a, b]\right\}$ and $\mathrm{Cl}(Q(L))=\{\mathrm{Cl}(A) \mid A \subseteq Q(L)\}$.

The following lemma is straightforward.

Lemma 1. Let $L$ be a lattice of finite length and $A, B \subseteq Q(L)$. Then

(i) $\bigcup_{A}[a, b]=\bigcup_{\mathrm{Cl}(A)}[x, y]$ and,

(ii) $\bigcup_{\mathrm{Cl}(A)}[x, y] \subseteq \bigcup_{\mathrm{Cl}(B)}[u, v]$ if and only if $\mathrm{Cl}(A) \subseteq \mathrm{Cl}(B)$.

The next lemma is an easy consequence of Lemma 1.

LEMma 2. Let $L$ be a lattice of finite length. Then

(i) $\mathrm{Cl}$ is a closure operator on $Q(L)$ and,

(ii) $\mathrm{Cl}(Q(L))$ is a lattice with respect to set inclusion. 
THEOREM 1. For a lattice $L$ of finite length the following conditions are equivalent:

(i) L is distributive;

(ii) $L-\bigcup_{A}[a, b]$ is a sublattice of $L$ for every $A \subseteq Q(L)$;

(iii) for every $S \subseteq L, S$ is a sublattice of $L$ if and only if $S=L-\bigcup_{A}[a, b]$ for some $A \subseteq Q(L)$;

(iv) the mapping $\varphi(S)=\mathrm{Cl}(A)$, where $S=L-\bigcup_{A}[a, b], A \subseteq Q(L)$, is an isomorphism between $\operatorname{Sub}(L)$ and the dual of $\mathrm{Cl}(Q(L))$.

Proof. That (i) implies (ii) follows from the fact that join-irreducible elements in a distributive lattice are join-prime, that is, if $a \in J(L)$ and $a \leqq b \vee c$ then $a \leqq b$ or $a \leqq c$. Applying Proposition 1 we get that (ii) implies (iii). On the other hand, Proposition 1 together with Lemma 1(ii) shows that $\varphi$ is well-defined, one-one, isotone, and that, in fact, $\varphi^{-1}$ is isotone. From (iii) we have that $\varphi$ is onto, so that $\varphi$ is, indeed, an isomorphism; thus, (iii) implies (iv). It remains only to show that (iv) implies (i).
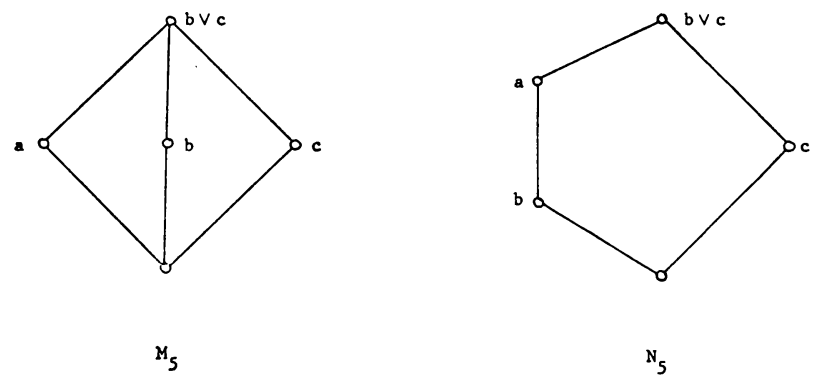

Figure 1

Let $M_{5}$ and $N_{5}$ be the two five-element nondistributive lattices labelled as in Figure 1. Suppose that $L$ satisfies (iv) but $L$ is nondistributive. Then $L$ contains as a sublattice a copy of $M_{5}$ or $N_{5}$. Let $d$ be a join-irreducible in $L$ such that $d \leqq a$ but $d \leq b \wedge c$, and $e$ a meet-irreducible in $L$ such that $e \geqq b \vee c$. By the surjectivity of $\varphi^{-1}, L-\bigcup_{\mathrm{Cl}(\{e / d\})}[x, y]$ is a sublattice of $L$. In view of Lemma $1(\mathrm{i}), L-\bigcup_{\mathrm{Cl}(\{e / d\})}[x, y]=L-[d, e]$. But $b \vee c \in[d, e]$ although $b, c \in L-[d, e]$, which is a contradiction. Thus, (iv) implies (i), completing the proof.

3. Maximal proper sublattices of finite distributive lattices. We define a partial ordering on $Q(L)$ as follows: $b / a \leqq d / c$ if and only if $[a, b] \subseteq[c, d]$. If $b / a$ is minimal with respect to this ordering then $\operatorname{Cl}(\{b / a\})=\{b / a\}$ so that by Theorem $1, L-[a, b]$ is a maximal proper sublattice of $L$ in the case that $L$ is finite distributive. Note that if $b / a \in Q(L)$ then $b / a$ is minimal if and only if $[a, b) \subseteq L-M(L)$ and $(a, b] \subseteq L-J(L)$ (cf. [2, Theorem 3]). 
For $x, y \in L, x$ covers $y(x>y$ or $y<x)$ in $L$ if $x>y$ and $x \geqq z>y$ implies $x=z$, for every $z \in L$. For $A \subseteq L$ we define $\operatorname{cov}(A)=\{x \in L \mid x>a$ or $x<a$ or $x=a$, for some $a \in A\}$. Observe that $a \in L-J(L)(a \in L-M(L))$ if and only if there exist $b, c \in \operatorname{cov}(\{a\})$ such that $a=b \vee c(a=b \wedge c)$.

THEOREM 2. Let $L$ be a finite distributive lattice and $K=L-[a, b]$ (b/a $\in Q(L), a \neq b)$ be a maximal proper sublattice of $L$. Then (i) $\operatorname{cov}([a, b])$ is a sublattice of $L$ isomorphic to the direct product of $[a, b]$ with a threeelement chain, and (ii) $J(K)=(J(L)-\{a\}) \cup\{c\}$, where $a<c \in K$.

Proof. Set

$$
\begin{aligned}
A & =\{y \in K \mid y<x \text { for some } x \in[a, b]\}, \\
B & =\{y \in K \mid y>x \text { for some } x \in[a, b]\}, \\
A^{\prime} & =\{x \in[a, b] \mid x>y \text { for some } y \in A\}, \\
B^{\prime} & =\{x \in[a, b] \mid x<y \text { for some } y \in B\} .
\end{aligned}
$$

To establish (i) it suffices to show that $A \cong[a, b] \cong B$. Since $b / a$ is minimal in $Q(L)$ and $a \neq b$, it follows that $a \neq 0$ and $b \neq 1$; thus, $a \in A^{\prime}$ and $b \in B^{\prime}$. Furthermore, since $L-[a, b]$ is a sublattice of $L$, every element in $A^{\prime}$ covers precisely one element in $A$ and every element in $B^{\prime}$ is covered by precisely one element in $B$.

Suppose now that $c_{1}^{\prime}, c_{2}^{\prime}$ are distinct minimal elements in $B^{\prime}$ with covers $c_{1}, c_{2} \in B$. Since $[a, b]$ is a sublattice of $L, c_{1} \neq c_{2}$; since $c_{1}^{\prime}$ is incomparable with $c_{2}^{\prime}, c_{1}$ is incomparable with $c_{2}$; and since $L-[a, b]$ is a sublattice of $L$, $c_{1}, c_{2}>c_{1} \wedge c_{2} \in L-[a, b]$. Now, if $c_{2}^{\prime}=c_{2}^{\prime} \vee\left(c_{1} \wedge c_{2}\right)$ then $c_{1}^{\prime} \wedge c_{2}^{\prime}<c_{1} \wedge c_{2}<c_{2}^{\prime}$, so that $c_{1} \wedge c_{2} \in[a, b]$. Therefore, $c_{2}^{\prime}<c_{2}^{\prime} \vee\left(c_{1} \wedge c_{2}\right) \leqq c_{2}$ and, since $c_{2}^{\prime}<c_{2}$, we have that $c_{2}^{\prime}\left\langle c_{2}^{\prime} \vee\left(c_{1} \wedge c_{2}\right)=c_{2}\right.$ which, by transposition implies that $c_{2}^{\prime} \wedge\left(c_{1} \wedge c_{2}\right)<c_{1} \wedge c_{2}$. But $c_{1}^{\prime} \wedge c_{2}^{\prime} \leqq c_{2}^{\prime} \wedge\left(c_{1} \wedge c_{2}\right)<c_{2}^{\prime}$ so that $c_{2}^{\prime} \wedge\left(c_{1} \wedge c_{2}\right) \in B^{\prime}$, contradicting the minimality of $c_{2}^{\prime}$. Thus, $B^{\prime}$ has a unique minimal element $c^{\prime}$ with precisely one cover $c$ in $B$; dually, $A^{\prime}$ has a unique maximal element $d^{\prime}$ covering precisely one element $d$ in $A$. Now, if $f$ is the unique cover of $b$ and $e$ the unique element covered by $a$ then by transposition we have that $A=[e, d] \cong\left[a, d^{\prime}\right]=A^{\prime}$ and $B=[c, f] \cong\left[c^{\prime}, b\right]=B^{\prime}$. From this it follows that $\operatorname{cov}([a, b])=A \cup[a, b] \cup B$ is a sublattice of $L$ and that in fact, $b / a$ is minimal in $Q(\operatorname{cov}([a, b]))$. In this case $A \cup B$ is a maximal proper sublattice of $\operatorname{cov}([a, b])$ so that by $[2$, Theorem 2$],|A \cup B| \geqq \frac{2}{3}|\operatorname{cov}([a, b])|$. Now, if $d<b$ or $a<c$ then $|\operatorname{cov}([a, b])|=|A|+|[a, b]|+|B|<3|[a, b]|$. But $[a, b]=\operatorname{cov}([a, b])-(A \cup B)$ so that $|A \cup B|<\frac{2}{3}|\operatorname{cov}([a, b])|$, which is a contradiction. Thus, $a=c^{\prime}$ and $b=d^{\prime}$ so that $A \cong[a, b] \cong B$, from which (i) follows.

To show (ii) observe first that $J(L)-\{a\} \subseteq J(K)$ and $J(K) \cap A \subseteq J(L)-\{a\}$. It suffices then to show that $J(K) \cap B=\{c\}$. 
Let $x \in B-\{c\}$. Choose some $y \in B$ such that $x>y$. Then there exist $x_{1}, y_{1} \in[a, b]$ and $x_{2}, y_{2} \in A$ such that $x>x_{1}>x_{2}$ and $y>y_{1}>y_{2}$. By transposition $x_{1}>y_{1}, x_{2}>y_{2}$, and $x_{1} \wedge y=y_{1}$. If $x_{2}<y$ then $y_{1}=x_{1} \wedge y \geqq x_{2}>y_{2}$, and since $y_{1}>y_{2}$ we have that $y_{1}=x_{2}$, which is impossible. Thus, $x_{2}$ is incomparable with $y$ and, in fact, $x$ covers $x_{2}$ in $K$, and since $x$ also covers $y$ in $K$, we get that $x$ is join-reducible in $K$.

It remains only to show that $c \in J(K)$. We may without loss of generality assume that $c$ covers two distinct incomparable elements $c_{1}, c_{2} \in L$, both incomparable with $a$. But $a$ is join-irreducible in $L$, that is, it covers only $e$. By transposition we get that $\left\{a, c_{1}, c_{2}, e, c\right\}$ is a sublattice of $L$ isomorphic to the five-element modular, nondistributive lattice $M_{5}$ which, of course, is a contradiction. The proof of the theorem is now complete.

The following corollary is an immediate consequence of Theorem 2(i).

COROLlARY 1. Every distributive lattice of order $n \geqq 3$ which contains a maximal proper sublattice of order $m$ also contains sublattices of orders $n-m, 2(n-m)$, and $3(n-m)$.

COROLlaRy 2. Every finite distributive lattice $L$ contains a maximal proper sublattice $K$ such that either $|K|=|L|-1$ or $|K| \geqq 2 l(L)$.

Proof. We may without loss of generality assume that $\operatorname{Irr}(L)=\varnothing$. Recall that for finite distributive lattices $|J(L)|=l(L)+1=|M(L)|$. Furthermore, the inequality $|L| \geqq|J(L)|+|M(L)|-|\operatorname{Irr}(L)|$ holds in every lattice $L$ of finite length, so that if $L$ is distributive we have that $|L| \geqq$ $2(l(L)+1)-|\operatorname{Irr}(L)|$. (This latter inequality, incidentally, holds in every lattice of finite length, cf. [3, Theorem 1].)

If $J(K)=J(L)-\{a\}$ then $M(K)=M(L)-\{b\}$, and since $J(L) \cap M(L)=$ $\operatorname{Irr}(L)=\varnothing$ we also have that $\operatorname{Irr}(K)=\varnothing$. In this case $|K| \geqq 2(l(K)+1)-$ $|\operatorname{Irr}(K)|=2|J(L)-\{a\}|=2 l(L)$.

Otherwise, $J(K) \neq J(L)-\{a\}$. By Theorem 2(ii) and its dual there exist $c, d \in L$ such that $J(K)=(J(L)-\{a\}) \cup\{c\}, c \notin J(L)$, and $M(K(=(M(L)-$ $\{b\}) \cup\{d\}, d \notin M(L)$. Observe that $(J(L)-\{a\}) \cap(M(L)-\{b\}) \subseteq J(L) \cap$ $M(L)=\operatorname{Irr}(L)=\varnothing$. Therefore, $\operatorname{Irr}(K) \subseteq\{c, d\}$, so that in this case

$$
\begin{aligned}
|K| & \geqq 2(l(K)+1)-|\operatorname{Irr}(K)| \\
& \geqq 2(|(J(L)-\{a\}) \cup\{c\}|+1)-2=2 l(L) .
\end{aligned}
$$

The estimate on the order of maximal proper sublattices of finite distributive lattices prescribed in Corollary 2 is best possible in the sense that, if for every positive integer $n, L_{n}$ is the ordinal sum of $n$ copies of the Boolean lattice $2^{3}$, then the maximum order of a maximal proper sublattice of $L_{n}$ is $2 l\left(L_{n}\right)$. 


\section{REFERENCES}

1. G. Birkhoff, Lattice theory, 3rd ed., Amer. Math. Soc. Colloq. Publ., vol. 25, Amer. Math. Soc., Providence, R.I., 1967. MR 37 \#2638.

2. I. Rival, Maximal sublattices of finite distributive lattices, Proc. Amer. Math. Soc. 37 (1973), 417-420.

3. - Lattices with doubly irreducible elements, Canad. Math. Bull. (to appear).

Department of Mathematics, University of Manitoba, Winnipeg, Manitoba R3T 2N2, CANADA 